\title{
Finite-time synchronization criterion of graph theory perspective fractional-order coupled discontinuous neural networks
}

\author{
A. Pratap ${ }^{1}$, R. Raja ${ }^{2}$, Jinde Cao ${ }^{3 *}$, J. Alzabut ${ }^{4}$ and Chuangxia Huang ${ }^{5^{*}}$ (D)
}

\section{"Correspondence:}

jdcao@seu.edu.cn;

cxiahuang@126.com

${ }^{3}$ School of Mathematics, Southeast

University, Nanjing, China

${ }^{5}$ School of Mathematics and

Statistics, and Hunan Provincial Key Laboratory of Mathematical

Modeling and Analysis in

Engineering, Changsha University of

Science and Technology, Changsha,

China

Full list of author information is

available at the end of the article

\section{Springer}

\begin{abstract}
In this research work, the finite-time synchronization and adaptive finite-time synchronization criterion of graph theory perspective fractional-order coupled discontinuous neural networks (FCDNNs) are investigated under two different control strategies. By utilizing differential inclusion theory, Filippov framework, suitable Lyapunov functional, and graph theory approach, several sufficient criteria based on discontinuous state feedback control protocol and discontinuous adaptive feedback control protocol are established for ensuring the finite-time synchronization and adaptive finite-time synchronization of FCDNNs. Finally, two numerical cases illustrate the efficiency of the proposed finite-time synchronization results.
\end{abstract}

Keywords: Discontinuous fractional-order neural networks; Coupled systems; Finite time synchronization

\section{Introduction and modeling}

In recent times, differential equation and fractional differential equation models have found their applications in a variety of fields including biology [1-6], physics [7-10], engineering [11-14], mathematics [15-18], information technology, and so on [19-27]. They are also one of the most rudimentary tools for neural networks. They were first and foremost conferred by de Leibnitz and Gottfried Wilhelm Leibnitz in 1695 (see [28, 29]). Currently fractional-order calculus (FOC) has been considered predominantly due to its extensive applications in several fields, for instance, biology, control, optics, viscoelasticity, and signal processing (see [30-34]). As is known, FOC is an expansion of conventional integer-order calculus. At present, fractional-order differential techniques are employed widely to explore the dynamical behaviors of the networks, especially neural networks $(\mathrm{NNs})$ and complex networks (CNs) (see [35-38]). The fractional-order differential systems possess unlimited memory property and more degrees of freedom in contrast to the conventional integer-order differential systems, which is the main benefit of the FOC. As a consequence of these benefits, some researchers have shown their keen interest to integrate the FOC into NNs to make fractional-order neural networks (FONNs) models. Among others, the dynamical behaviors of FONNs have already become a hot research topic, and lots of scientific results have been well published in the literature (see [39-43]).

(c) The Author(s) 2020. This article is licensed under a Creative Commons Attribution 4.0 International License, which permits use sharing, adaptation, distribution and reproduction in any medium or format, as long as you give appropriate credit to the original author(s) and the source, provide a link to the Creative Commons licence, and indicate if changes were made. The images or other third party material in this article are included in the article's Creative Commons licence, unless indicated otherwise in a credit line to the material. If material is not included in the article's Creative Commons licence and your intended use is not permitted by statutory regulation or exceeds the permitted use, you will need to obtain permission directly from the copyright holder. To view a copy of this licence, visit http://creativecommons.org/licenses/by/4.0/. 
Synchronization, which defines the dynamical behaviors of coupled systems achieving the same spatial state at the same time, has become an important research topic, and it has been successfully applied in image processing, secure communication, optimization, and so on. The synchronization is majorly segregated into two classifications based on the existing synchronization results and convergence time such as infinite-time synchronization (IFTS), exponential synchronization, lag synchronization, asymptotical synchronization and Mittag-Leffler synchronization, and finite-time synchronization (FTS). Generally, asymptotic synchronization reaches an infinite time, it becomes infinite synchronization. In realistic engineering applications, humans continuously like to obtain synchronization in a finite convergence time, which is known as FTS. Moreover, time delays are inevitable in nearly all dynamical systems including neural network, chemical process, and nuclear reactors, which may lead to system oscillation, instability behaviors, and divergence because of the limited switching speed of amplifier circuits (see [44-48]). In recent decades, an increasing interest in the field of finite-time synchronization criterion of FONNs with time delays has attracted many scientific communities, which has given rise to some meaningful and significant outcomes (see [49-51]).

During the last decades, complex dynamical networks (CDNs) have obtained the attention of many researchers owing to their wide application in diverse areas like global economic markets, traffic control networks, communication networks, and synchronization results have been discussed in the existing literature (see $[37,38,52,53])$. Coupled neural networks (CONNs) can be recognized as a significant improvement of complex dynamical networks $[54,55]$. In CONNs, the coupling term depends on the following perspectives: (1) The relation and control between at least two neurons (see [56, 57]); (2) The amount of information that transfers from one neuron to another neuron (see [58]); (3) The complex structure between at least two neurons (see [59]). CONNs have been applied in numerous fields like harmonic oscillation generation, image encryption, and classification (see $[60,61])$. In recent years, some significant related results of the fractional-order CONNs can be witnessed in the previous literature (see $[62,63])$. Even though there exist numerous fractional-order CONNs, so far, most of them have considered the case of continuous neuron activations only. But in general, signal transmission between neurons and signal output of neuron are all discontinuous. The activation functions of CONNs are not generally continuous. As a result, the classical solution for fractional-order differential equations is not suitable to consider FCDNNs. Thus the investigation of FCDNNs is significant and much more challenging. Unfortunately, according to our literature survey, no one has investigated the FTS and adaptive FTS criterion of graph theory perspective FCDNNs. This situation stimulates the interest towards the further investigation for FCDNNs.

Motivated by the above conversation, we aim to analyze the FTS and adaptive FTS criterion of graph theory perspective FCDNNs with time delays using graph theory techniques. The main novelty of this research work is outlined in detail as follows:

1. For the first time the algebraic graph theory technique is incorporated into FTS and adaptive FTS criterion of FCDNNs with time delays.

2. These theoretical results and techniques can be extended to FTS of fractional-order CONNs and fractional-order discontinuous neural networks.

3. Two kinds of different control strategies, such as discontinuous state feedback control and discontinuous adaptive feedback control, are designed respectively to achieve the FTS and adaptive FTS of a class of FCDNNs. 
4. By using the discontinuous FONNs results, coupling terms are added to discontinuous FONNs, and these results are established by using graph theoretical concepts.

5. Moreover, the proposed results in this paper are also still valid for FTS and adaptive FTS criterion for both integer-order and fractional-order CONNs with continuous activations, respectively, and these results do not exist in the previous works of literature.

Section 2 contains basic results on a graph-theoretical concept, fractional-order calculus, and formulations for FCDNNs systems. Section 3 is formulated for two different control strategies. Here we also derive sufficient criteria for the FTS and adaptive FTS criterion of FCDNNs based on algebraic graph theory techniques. Two numerical cases with simulations are established to demonstrate the efficiency of the obtained synchronization results in Sect. 4. At last, Sect. 5 terminates with conclusions.

\section{Basic knowledge and model description}

In this section, some basic concepts of graph theoretical results, fractional-order calculus, problem statement, and some necessary assumptions are given.

A directed graph $\mathcal{G}=(\mathcal{V}, \mathcal{E})$ consists of vertices or nodes $\mathcal{V}=\{1,2, \ldots, N\}$ and a set $\mathcal{E}$ of $\operatorname{arcs}(k, l)$ leading from $k$ th node to $l$ th node. A directed path $\mathbb{D}$ is a subgraph of $\mathcal{G}$ with distinct vertices $\{1,2, \ldots, p\}$ such that its set of $\operatorname{arcs}$ is $\left\{\left(k_{x}, k_{x+1}\right): x=1,2, \ldots, p-1\right\}$. If the first and last nodes are similar, $\mathbb{D}$ is a directed cycle. A graph is strongly connected if there exists a directed path from $k$ to $l$ in $\mathcal{G}$. A graph $\mathcal{G}$ with weight matrix $A=\left(a_{j k}\right)_{N \times N}$ is represented as $(\mathcal{G}, A)$, here $a_{j k}>0$ equals the weight of arc $(j, k)$ if it exists, and zero otherwise. The Laplacian matrix of $(\mathcal{G}, A)$ is described as follows:

$$
\mathcal{L}=\left(l_{j k}\right)_{N \times N}= \begin{cases}-a_{j k} & \text { if } j \neq k, \\ \sum_{j \neq p} a_{j p} & \text { if } j=k .\end{cases}
$$

Lemma 2.1 ([64]) Let the kth diagonal element of cofactor of the Laplacian matrix of $(\mathcal{G}, A)$ be represented by $\gamma_{k}$ and assume $n \geq 2$. Then the following relationship holds:

$$
\sum_{k, l}^{N} \gamma_{k} a_{k l} \Omega_{k l}\left(q_{k}, q_{l}\right)=\sum_{B \in \mathcal{B}} \mathcal{W}(B) \sum_{(i, m) \in \mathcal{E}\left(\mathcal{A}_{B}\right)} \Omega_{m, i}\left(q_{m}, q_{i}\right),
$$

where $\Omega_{k i}\left(q_{k}, q_{i}\right), k, i \in\{1,2, \ldots, N\}$ are arbitrary functions, $\mathcal{B}$ denotes the set of all spanning unicyclic graphs of $(\mathcal{G}, A), \mathcal{W}(B)$ and $\mathcal{A}_{B}$ respectively the weights and directed cycle of $B$. Moreover, $\gamma_{k}>0$ if $(\mathcal{G}, A)$ is strongly connected for $k \in\{1,2, \ldots, N\}$.

In order to describe our system, some basic definitions and important lemmas with respect to fractional-order calculus are presented.

Definition 2.2 $([29,65])$ The fractional-order integral of $q(t)$ is described as follows:

$$
D^{-h} q(t)=\frac{1}{\Gamma(h)} \int_{0}^{t}(t-\varsigma)^{h-1} y(\varsigma) \mathrm{d} \varsigma
$$

where $h>0$ and $\Gamma(\cdot)$ is the gamma function. 
Definition $2.3([29,65])$ The Caputo fractional integral of $q(t)$ is described as follows:

$$
D^{h} q(t)= \begin{cases}D^{-(n-h)}\left(\frac{d^{n}}{d t^{n}} q(t)\right) & \text { if } h \in(n-1, n) \\ \left(\frac{d^{n}}{d t^{n}} q(t)\right) & \text { if } h=n,\end{cases}
$$

where $h \in \mathbb{R}^{+}, n \in \mathbb{Z}^{+}$.

In this paper, we consider an array of fractional-order coupled discontinuous neural networks (FCDNNs) consisting of $N$ identical nodes with each isolated node network being an $n$-dimensional dynamical system, which is presented by

$$
\begin{aligned}
D^{h} p_{k}(t)= & -U p_{k}(t)+V f\left(p_{k}(t)\right) \\
& +W f\left(p_{k}(t-\tau)\right)+\sum_{l=1}^{N} a_{k l} E\left(q_{l}(t)-q_{k}(t)\right)+y_{k}(t),
\end{aligned}
$$

in which $k=1,2, \ldots, N, \mathrm{~N}$ is the total number of nodes in the networks, $D^{h}$ signifies the Caputo fractional-order derivative with order $0<h<1, p_{k}(t)=\left(p_{k 1}(t), p_{k 2}(t), \ldots, p_{k n}(t)\right)^{T}$ is the state vector of the $k t h$ dynamical node, $U=\operatorname{diag}\left\{u_{1}, \ldots, u_{n}\right\}$ is the self inhibition, $y_{k}(t)=$ $\left(y_{k 1}(t), y_{k 2}(t), \ldots, y_{k n}(t)\right)^{T}$ is the control inputs, $f\left(p_{k}(t)\right)=\left(f_{1}\left(p_{k 1}(t)\right), \ldots, f_{n}\left(p_{k n}(t)\right)\right)^{T}$ signifies activation functions of the neurons at time $t, \tau$ is a positive constant, and $V=\left[v_{k l}\right]_{n \times n}$ and $W=\left[w_{k l}\right]_{n \times n}$ represent the connection weights of the $k t h$ neuron to lth neuron, $E=$ $\operatorname{diag}\left\{e_{1}, \ldots, e_{n}\right\}$ represents the inner coupling matrices between two nodes $k$ and $l$ at the time $t$, and $A=\left(a_{k l}\right)_{N \times N}$ is the topological structure of the networks (1), in which $a_{k l}$ is defined as follows: if there are links from node $k$ to node $l(k \neq l)$, then $a_{k l}>0$, otherwise $a_{k l}=0$, and we assume that $a_{k k}=0, k \in\{1,2, \ldots, N\}$.

Let $q(t)$ be an isolated node, the dynamics of which is given by

$$
D^{h} q(t)=-U q(t)+V f(q(t))+W f(q(t-\tau))
$$

Since the neuron activation function $f(\cdot)$ is a discontinuous function, the traditional solutions are not suitable to considered system (1) and (2). To this conclusion, we introduce the concept of Filippov solution (see [66]).

Our main aim is to solve the synchronization problem of FCDNNs. Before that, we make the following definitions, assumptions, and lemmas.

Definition 2.4 A vector-valued function $p(t)$ is said to be a Filippov solution for fractional differential systems $D^{h} p(t)=f(t, p)$, and it is defined on a degenerate interval $\mathcal{I} \subseteq \mathbb{R}$. The Filippov solution for fractional differential systems with initial values $p(0)=p_{0}$ is absolutely continuous on any compact subinterval $\left[t_{1}, t_{2}\right]$ of $\mathcal{I}$ and for almost all $t \in \mathcal{I}$. In addition, $p(0)=p_{0}$ and functional differential inclusions

$$
D^{h} p(t) \in \mathbb{F}(t, p),
$$

where $\mathbb{F}(t, p)$ is the set-valued map of $f(t, p)$, hold. 
Suppose that the neuron activation function $f(\cdot)$ satisfies the following conditions.

Assumption $\left[\mathcal{H}_{1}\right]$. For every $k=1,2, \ldots, n$, suppose that the discontinuous activations $f_{k}: \mathbb{R} \rightarrow \mathbb{R}$ are bounded and continuous functions except on a finite number of jump discontinuities $\sigma_{\iota}$ on every bounded interval. Furthermore, there exist left limits $f_{k}\left(\sigma_{\imath}^{-}\right)$and right limits $f_{k}\left(\sigma_{l}^{+}\right)$, respectively.

Based on Definition 2.4, if the activation function satisfies Assumption $\left[\mathcal{H}_{1}\right]$, one can obtain that

$$
\begin{aligned}
& \mathbb{F}\left[p_{k}(t)\right]=\tilde{c o}\left[f\left(p_{k}(t)\right)\right]=\left(\tilde{c o}\left[f_{k 1}\left(p_{k 1}(t)\right)\right], \ldots, \tilde{c o}\left[f_{k n}\left(p_{k n}(t)\right)\right]\right)^{T} \text { and } \\
& \tilde{c o}\left[f_{l}\left(p_{k l}(t)\right)\right]=\left[\min \left\{\tilde{c o}\left[f_{l}\left(p_{k l}^{-}(t)\right)\right], \tilde{c o}\left[f_{l}\left(p_{k l}^{+}(t)\right)\right]\right\}, \max \left\{\tilde{c o}\left[f_{l}\left(p_{k l}^{-}(t)\right)\right], \tilde{c o}\left[f_{l}\left(p_{k l}^{+}(t)\right)\right]\right\}\right] .
\end{aligned}
$$

Assumption $\left[\mathcal{H}_{2}\right]$. For every $k=1,2, \ldots, N, l=1,2, \ldots, n$, there exist positive constants $b_{l}, d_{l}>0$ such that, for every $p_{k l}(t) \in \mathbb{R}, q_{l}(t) \in \mathbb{R}, \tilde{\mu}_{l}(t) \in \tilde{c o}\left[f_{l}\left(p_{k l}(t)\right)\right]$, and $\mu_{l}(t) \in$ $\tilde{c o}\left[f_{l}\left(q_{l}(t)\right)\right]$, the following inequality holds:

$$
\left|\tilde{\mu}_{k l}(t)-\mu_{l}(t)\right| \leq b_{l}\left|p_{k l}(t)-q_{l}(t)\right|+d_{l}
$$

Furthermore, $0 \in \tilde{c o}\left[f_{l}(0)\right]$.

Define the error signal: $\alpha_{k}(t)=p_{k}(t)-q(t)$, then the synchronization error system can be obtained from (1) and (2) as follows:

$$
\begin{aligned}
D^{h} \alpha_{k}(t)= & -U \alpha_{k}(t)+V \Psi\left(\alpha_{k}(t)\right) \\
& +W \Psi\left(\alpha_{k}(t-\tau)\right)+\sum_{l=1}^{N} a_{k l} E\left(\alpha_{l}(t)-\alpha_{k}(t)\right)+y_{k}(t),
\end{aligned}
$$

where $\Psi\left(\alpha_{k}(t)\right)=f\left(p_{k}(t)\right)-f(q(t)), k=1,2, \ldots, N$. Let the initial values of error system (3) be given as

$$
\alpha_{k}(t)=\psi_{k}(t) \in \mathcal{C}\left([-\tau, 0], \mathbb{R}^{n}\right), \quad k=1,2, \ldots, N,
$$

where $\mathcal{C}\left([-\tau, 0], \mathbb{R}^{n}\right)$ represents the set of all continuous differential functions from $[-\tau, 0]$ into $\mathbb{R}^{n}$.

Definition 2.5 FCDNNs (1) is said to be finite-time synchronized with isolated networks (2) if there exists a settling time $t_{1}>0$, which is a real number if

$$
\lim _{t \rightarrow t_{1}}\left\|p_{k}(t)-q(t)\right\|=0 \quad \text { and } \quad\left\|p_{k}(t)-q(t)\right\|=0 \quad \text { for } t>t_{1}, k \in\{1,2, \ldots, N\}
$$

Lemma 2.6 ([67]) Let $q(t) \in \mathbb{R}^{n}$ be a continuously derivable vector-valued function, then

$$
D^{h}\left\{q^{T}(t) q(t)\right\} \leq 2 q^{T}(t)\left\{D^{h} q(t)\right\}, \quad h \in(0,1) .
$$

Lemma 2.7 ([68]) If $\Phi_{1}, \ldots, \Phi_{n} \geq 0,0<\vartheta<\epsilon$, then the following inequality is established:

$$
\left[\sum_{i=1}^{n} \Phi_{i}^{\epsilon}\right]^{\frac{1}{\epsilon}} \leq\left[\sum_{i=1}^{n} \Phi_{i}^{\vartheta}\right]^{\frac{1}{\vartheta}} .
$$


Lemma 2.8 ([52]) Assume that the positive definite function $X(t)$ is a continuous function, and it satisfies the following differential inequality:

$$
D^{h} X(t) \leq-\delta X^{v}(t)
$$

where $\delta>0,0<v<h$ are all constants. Then $X(t)$ satisfies the following differential inequality:

$$
X^{h-v}(t) \leq X^{h-v}\left(t_{0}\right)-\frac{\delta \Gamma(1+h-v)\left(t-t_{0}\right)^{h}}{\Gamma(1+h) \Gamma(1-v)}, \quad t \in\left[t_{0}, t_{1}\right]
$$

and $X(t)=0, \forall t \geq t_{1}$, where $t_{1}$ is denoted by

$$
t_{1}=t_{0}+\left[\frac{\Gamma(1+h) \Gamma(1-v) X^{h-v}\left(t_{0}\right)}{\delta \Gamma(1+h-v)}\right]^{\frac{1}{h}} .
$$

Remark 2.9 In $[69,70]$, the authors demonstrated the synchronization criterion of discontinuous fractional-order neural networks by using state feedback control law. The global synchronization criterion of FOCDNs by using graph theory techniques was analyzed in [53]. In [62, 63], the authors investigated the synchronization criterion of FOCONNs by using the LMI technique and Kronecker product technique. Besides, it is helpful for us to demonstrate our required finite-time synchronization and adaptive finitetime synchronization criterion from the results obtained in the aforementioned references $[53,62,63,69,70]$.

\section{Main results}

In this section, we demonstrate the finite-time synchronization and adaptive finite-time synchronization criterion of FCDNNs (1) and the isolated networks (2) by using graph theory techniques, discontinuous state feedback control, and discontinuous adaptive feedback control.

\subsection{Finite-time synchronization under discontinuous state feedback control}

First, we design discontinuous feedback control protocol as follows:

$$
\begin{aligned}
y_{k}(t)= & -\beta \alpha_{k}(t)-\zeta\left\|\alpha_{k}(t-\tau)\right\|_{1} \operatorname{sign}\left(\alpha_{k}(t)\right) \\
& -\eta \operatorname{sign}\left(\alpha_{k}(t)\right)-\xi \operatorname{sign}\left(\alpha_{k}(t)\right)\left|\alpha_{k}(t)\right|^{\theta},
\end{aligned}
$$

where $0<\theta<1, \beta>0, \zeta>0, \eta>0, \xi>0$ are control gains, they are properly selected in the following main theorem. According to the Filippov framework, FCDNNs (3) can be written as follows:

$$
\begin{aligned}
D^{h} \alpha_{k}(t) \in & -U \alpha_{k}(t)+V \tilde{c o}\left\{\Psi\left(\alpha_{k}(t)\right)\right\}+W \tilde{C o}\left\{\Psi\left(\alpha_{k}(t-\tau)\right)\right\} \\
& +\sum_{l=1}^{N} a_{k l} E\left(\alpha_{l}(t)-\alpha_{k}(t)\right)-\beta \alpha_{k}(t) \\
& -\zeta\left\|\alpha_{k}(t-\tau)\right\|_{1} \tilde{c o}\left(\operatorname{sign}\left(\alpha_{k}(t)\right)\right)-\eta \tilde{c o}\left(\operatorname{sign}\left(\alpha_{k}(t)\right)\right)
\end{aligned}
$$




$$
\begin{aligned}
& -\xi \tilde{c o}\left(\operatorname{sign}\left(\alpha_{k}(t)\right)\right)\left|\alpha_{k}(t)\right|^{\theta} \\
\subseteq & -U \alpha_{k}(t)+V\left[\tilde{c o}\left(p_{k}(t)\right)-\tilde{c o}(q(t))\right] \\
& +W\left[\tilde{c o}\left(p_{k}(t-\tau)\right)-\tilde{c o}(q(t-\tau))\right] \\
& +\sum_{l=1}^{N} a_{k l} E\left(\alpha_{l}(t)-\alpha_{k}(t)\right) \\
& -\beta \alpha_{k}(t)-\zeta\left\|\alpha_{k}(t-\tau)\right\|_{1} \tilde{c o}\left(\operatorname{sign}\left(\alpha_{k}(t)\right)\right) \\
& -\eta \tilde{c o}\left(\operatorname{sign}\left(\alpha_{k}(t)\right)\right)-\xi \tilde{c o}\left(\operatorname{sign}\left(\alpha_{k}(t)\right)\right)\left|\alpha_{k}(t)\right|^{\theta} .
\end{aligned}
$$

Then there exist a measurable function $\tilde{\mu}_{k}(t) \in \tilde{c o}\left[f\left(p_{k}(t)\right)\right]$ and $\mu(t) \in \tilde{c o}[f(q(t))]$ for a.e. $t \in\left[-\tau, t_{1}\right]$ such that

$$
\begin{aligned}
D^{h} \alpha_{k}(t)= & -U \alpha_{k}(t)+V\left[\tilde{\mu}_{k}(t)-\mu(t)\right] \\
& +W\left[\tilde{\mu}_{k}(t-\tau)-\mu(t-\tau)\right]+\sum_{l=1}^{N} a_{k l} E\left(\alpha_{l}(t)-\alpha_{k}(t)\right) \\
& -\beta \alpha_{k}(t)-\zeta\left\|\alpha_{k}(t-\tau)\right\|_{1} \operatorname{SIGN}\left(\alpha_{k}(t)\right) \\
& -\eta \operatorname{SIGN}\left(\alpha_{k}(t)\right)-\xi \operatorname{SIGN}\left(\alpha_{k}(t-\tau)\right)\left|\alpha_{k}(t)\right|^{\theta},
\end{aligned}
$$

where $\operatorname{SIGN}\left(\alpha_{k}(t)\right)=\left[\operatorname{SIGN}\left(\alpha_{k 1}(t)\right), \ldots, \operatorname{SIGN}\left(\alpha_{k n}(t)\right)\right]^{T}$ with

$$
\operatorname{SIGN}(\alpha)= \begin{cases}-1, & \alpha<0 \\ {[-1,1],} & \alpha=0, \\ 1, & \alpha>0\end{cases}
$$

Before starting the finite-time synchronization results, we introduce the following notations: $b^{\max }=\max _{1 \leq l \leq n} b_{l}, d^{\max }=\max _{1 \leq l \leq n} d_{l}, v^{\max }=\max _{1 \leq l, j \leq n}\left|v_{l j}\right|, w^{\max }=\max _{1 \leq l, j \leq n}\left|w_{l j}\right|$, and $\widehat{\gamma}_{\min }=\min _{1 \leq k \leq N} \gamma_{k}$.

Theorem 3.1 Suppose that Assumptions $\left[\mathcal{H}_{1}\right]-\left[\mathcal{H}_{3}\right]$ hold. Then FCDNNs (1) and the isolated networks (2) are finite-time synchronized under the discontinuous feedback control protocol (4), if

$$
\begin{aligned}
& \beta>\Lambda_{\min }(U)-n b^{\max } v^{\max }, \\
& \zeta>b^{\max } w^{\max }, \\
& \eta>n d^{\max }\left(v^{\max }+w^{\max }\right) .
\end{aligned}
$$

Furthermore, the settling time is evaluated as follows:

$$
t_{1}=t_{0}+\left[\frac{\Gamma(1+h) \Gamma\left(\frac{1-\theta}{2}\right) X^{\frac{2 h-1-\theta}{2}}\left(t_{0}\right)}{\delta \Gamma\left(\frac{2 h+1-\theta}{2}\right)}\right]^{\frac{1}{h}},
$$

where $\delta=\xi 2^{\frac{\theta+1}{2}} \widehat{\gamma}_{\min }^{\frac{1-\theta}{2}}, X\left(t_{0}\right)=\sum_{k=1}^{N} \frac{\gamma_{k}}{2} \alpha_{k}^{T}\left(t_{0}\right) \alpha_{k}\left(t_{0}\right), \gamma_{k}$ signifies the cofactor of the ith diagonal elements of $\mathcal{L}$, and $\Lambda_{\min }(U)$ signifies the minimal eigenvalue of $U$. 
Proof For the FCDNNs error system (6), construct the following Lyapunov functional:

$$
X(t)=\sum_{k=1}^{N} \frac{\gamma_{k}}{2} \alpha_{k}^{T}(t) \alpha_{k}(t) .
$$

Since $(\mathcal{G}, A)$ is strongly connected, by using Lemma 2.1 , we get that $\gamma_{k}>0$ for $k \in$ $\{1,2, \ldots, N\}$. According to Lemma 2.6, one can get

$$
\begin{aligned}
D^{h} X(t) \leq & \sum_{k=1}^{N} \gamma_{k} \alpha_{k}^{T}(t) D^{h}\left\{\alpha_{k}(t)\right\} \\
= & \sum_{k=1}^{N} \gamma_{k} \alpha_{k}^{T}(t)\left[-U \alpha_{k}(t)+V\left[\tilde{\mu}_{k}(t)-\mu(t)\right]\right. \\
& +W\left[\tilde{\mu}_{k}(t-\tau)-\mu(t-\tau)\right]+\sum_{l=1}^{N} a_{k l} E\left(\alpha_{l}(t)-\alpha_{k}(t)\right) \\
& -\beta \alpha_{k}(t)-\zeta\left\|\alpha_{k}(t-\tau)\right\|_{1} \operatorname{SIGN}\left(\alpha_{k}(t)\right) \\
& \left.-\eta \operatorname{SIGN}\left(\alpha_{k}(t)\right)-\xi \operatorname{SIGN}\left(\alpha_{k}(t)\right)\left|\alpha_{k}(t)\right|^{\theta}\right] .
\end{aligned}
$$

Based on Assumption $\left[\mathcal{H}_{2}\right]$, one has

$$
\begin{aligned}
\sum_{k=1}^{N} \gamma_{k} \alpha_{k}^{T}(t) V\left[\tilde{\mu}_{k}(t)-\mu(t)\right]= & \sum_{k=1}^{N} \sum_{l=1}^{n} \sum_{j=1}^{n} \gamma_{k} \alpha_{k l}(t) v_{l j}\left[\tilde{\mu}_{k j}(t)-\mu_{j}(t)\right] \\
\leq & \sum_{k=1}^{N} \sum_{l=1}^{n} \sum_{j=1}^{n} \gamma_{k}\left|\alpha_{k l}(t)\right|\left|v_{l j}\right|\left|\tilde{\mu}_{k j}(t)-\mu_{j}(t)\right| \\
\leq & \sum_{k=1}^{N} \sum_{l=1}^{n} \sum_{j=1}^{n} \gamma_{k}\left|\alpha_{k l}(t)\right|\left|v_{l j}\right|\left[b_{j}\left|\alpha_{k j}(t)\right|+d_{j}\right] \\
\leq & b^{\max } v^{\max } \sum_{k=1}^{N} \sum_{l=1}^{n} \sum_{j=1}^{n} \gamma_{k}\left|\alpha_{k l}(t)\right|\left|\alpha_{k j}(t)\right| \\
& +v^{\max } \sum_{k=1}^{N} \sum_{l=1}^{n} \sum_{j=1}^{n} \gamma_{k}\left|\alpha_{k l}(t)\right| d_{j} \\
\leq & n b^{\max } v^{\max } \sum_{k=1}^{N} \gamma_{k} \alpha_{k}^{T}(t) \alpha_{k}(t) \\
& +n v^{\max } d^{\max } \sum_{k=1}^{N} \gamma_{k}\left\|\alpha_{k}(t)\right\|
\end{aligned}
$$

Similar to (12), one has

$$
\sum_{k=1}^{N} \gamma_{k} \alpha_{k}^{T}(t) W\left[\tilde{\mu}_{k}(t-\tau)-\mu(t-\tau)\right]
$$




$$
\begin{aligned}
& =\sum_{k=1}^{N} \sum_{l=1}^{n} \sum_{j=1}^{n} \gamma_{k} \alpha_{k l}(t) w_{l j}\left[\tilde{\mu}_{k j}(t-\tau)-\mu_{j}(t-\tau)\right] \\
& \leq \sum_{k=1}^{N} \sum_{l=1}^{n} \sum_{j=1}^{n} \gamma_{k}\left|\alpha_{k l}(t)\right|\left|w_{l j}\right|\left|\tilde{\mu}_{k j}(t-\tau)-\mu_{j}(t-\tau)\right| \\
& \leq \sum_{k=1}^{N} \sum_{l=1}^{n} \sum_{j=1}^{n} \gamma_{k}\left|\alpha_{k l}(t)\right|\left|w_{l j}\right|\left[b_{j}\left|\alpha_{k j}(t-\tau)\right|+d_{j}\right] \\
& \leq b^{\max } w^{\max } \sum_{k=1}^{N} \sum_{l=1}^{n} \sum_{j=1}^{n} \gamma_{k}\left|\alpha_{k l}(t)\right|\left|\alpha_{k j}(t-\tau)\right| \\
& \quad+n d^{\max } w^{\max } \sum_{k=1}^{N} \sum_{l=1}^{n} \gamma_{k}\left|\alpha_{k l}(t)\right| \\
& \leq b^{\max } w^{\max } \sum_{k=1}^{N} \gamma_{k}\left\|\alpha_{k}(t)\right\|_{1}\left\|\alpha_{k}(t-\tau)\right\|_{1} \\
& \quad+n d^{\max } w^{\max } \sum_{k=1}^{N} \gamma_{k}\left\|\alpha_{k}(t)\right\|_{1} .
\end{aligned}
$$

From Eqs. (11)-(13), one has

$$
\begin{aligned}
D^{h} X(t) \leq & -\left[\Lambda_{\min }(U)+\beta-n b^{\max } v^{\max }\right] \sum_{k=1}^{N} \gamma_{k} \alpha_{k}^{T}(t) \alpha_{k}(t) \\
& -\left[\zeta-b^{\max } w^{\max }\right] \sum_{k=1}^{N} \gamma_{k}\left\|\alpha_{k}(t)\right\|_{1}\left\|\alpha_{k}(t-\tau)\right\|_{1} \\
& -\left[\eta-n d^{\max }\left(v^{\max }+w^{\max }\right)\right] \sum_{k=1}^{N} \gamma_{k}\left\|\alpha_{k}(t)\right\|_{1} \\
& +\sum_{k=1}^{N} \sum_{l=1}^{N} \gamma_{k} \alpha_{k}^{T}(t) a_{k l} E\left(\alpha_{l}(t)-\alpha_{k}(t)\right) \\
& -\xi \sum_{k=1}^{N} \sum_{l=1}^{n} \gamma_{k}\left|\alpha_{k l}(t)\right|^{\theta+1} .
\end{aligned}
$$

Next, our aim is to prove

$$
\sum_{k=1}^{N} \sum_{l=1}^{N} \gamma_{k} \alpha_{k}^{T}(t) a_{k l} E\left(\alpha_{l}(t)-\alpha_{k}(t)\right)-\xi \sum_{k=1}^{N} \sum_{l=1}^{n} \gamma_{k}\left|\alpha_{k l}(t)\right|^{\theta+1} \leq-\delta X^{\frac{1+\theta}{2}}(t) .
$$

On the one side, we show that

$$
\sum_{k=1}^{N} \sum_{l=1}^{N} \gamma_{k} \alpha_{k}^{T}(t) a_{k l} E\left(\alpha_{l}(t)-\alpha_{k}(t)\right) \leq 0
$$


To this conclusion, let $\tilde{\alpha}_{k}(t)=\sqrt{E} \alpha_{k}(t)$, then we can obtain from Lemma 2.1 that

$$
\begin{aligned}
& \sum_{k=1}^{N} \sum_{l=1}^{N} \gamma_{k} \alpha_{k}^{T}(t) a_{k l} E\left(\alpha_{l}(t)-\alpha_{k}(t)\right) \\
& \quad=\sum_{k=1}^{N} \sum_{l=1}^{N} \gamma_{k} a_{k l} \tilde{\alpha}_{k}^{T}(t)\left(\tilde{\alpha}_{l}(t)-\tilde{\alpha}_{k}(t)\right) \\
& \quad \leq \frac{1}{2} \sum_{k=1}^{N} \sum_{l=1}^{N} \gamma_{k} a_{k l}\left(\tilde{\alpha}_{l}^{T}(t) \tilde{\alpha}_{l}(t)-\tilde{\alpha}_{k}^{T}(t) \tilde{\alpha}_{k}(t)\right) \\
& \quad=\frac{1}{2} \sum_{B \in \mathcal{B}} \mathcal{W}(B) \sum_{(i, m) \in \mathcal{E}\left(\mathcal{A}_{B}\right)}\left(\tilde{\alpha}_{i}^{T}(t) \tilde{\alpha}_{i}(t)-\tilde{\alpha}_{m}^{T}(t) \tilde{\alpha}_{m}(t)\right) .
\end{aligned}
$$

For any directed cycle $\mathcal{A}_{B}$, the set $\mathcal{E}\left(\mathcal{A}_{B}\right)$ can be regarded as

$$
\mathcal{E}\left(\mathcal{A}_{B}\right)=\left\{\left(k_{x}, k_{x+1}\right) / x=1,2, \ldots, p-1, p \leq N, k_{p}=k_{1}\right\} .
$$

It follows from (18) that

$$
\begin{aligned}
& \sum_{(i, m) \in \mathcal{E}}\left(\mathcal{A}_{B}\right) \\
& \left.=\tilde{\alpha}_{k_{1}}^{T}(t) \tilde{\alpha}_{k_{1}}(t)-\tilde{\alpha}_{k_{2}}^{T}(t) \tilde{\alpha}_{k_{2}}(t)-\tilde{\alpha}_{m}^{T}(t) \tilde{\alpha}_{m}(t)\right) \\
& \quad+\tilde{\alpha}_{k_{2}}^{T}(t) \tilde{\alpha}_{k_{2}}(t)-\tilde{\alpha}_{k_{3}}^{T}(t) \tilde{\alpha}_{k_{3}}(t) \\
& \quad+\tilde{\alpha}_{k_{3}}^{T}(t) \tilde{\alpha}_{k_{3}}(t)-\cdots \\
& \quad+\tilde{\alpha}_{k_{p-1}}^{T}(t) \tilde{\alpha}_{k_{p-1}}(t)-\tilde{\alpha}_{k_{p}}^{T}(t) \tilde{\alpha}_{k_{p}}(t) \\
& \quad+\tilde{\alpha}_{k_{p}}^{T}(t) \tilde{\alpha}_{k_{p}}(t)-\tilde{\alpha}_{k_{1}}^{T}(t) \tilde{\alpha}_{k_{1}}(t) \\
& =0 .
\end{aligned}
$$

From (17) and (19), one obtains

$$
\sum_{k=1}^{N} \sum_{l=1}^{N} \gamma_{k} \alpha_{k}^{T}(t) a_{k l} E\left(\alpha_{l}(t)-\alpha_{k}(t)\right) \leq 0 .
$$

On the other side, we show that

$$
-\xi \sum_{k=1}^{N} \sum_{l=1}^{n} \gamma_{k}\left|\alpha_{k l}(t)\right|^{\theta+1} \leq-\delta X^{\frac{1+\theta}{2}}(t) .
$$

According to Lemma 2.7, one obtains

$$
\begin{aligned}
-\sum_{k=1}^{N} \sum_{l=1}^{n} \gamma_{k}\left|\alpha_{k l}(t)\right|^{\theta+1} & =-\sum_{k=1}^{N} \sum_{l=1}^{n}\left[\gamma_{k}^{\frac{2}{1+\theta}}\left|\alpha_{k l}(t)\right|^{2}\right]^{\frac{\theta+1}{2}} \\
& \leq-\sum_{k=1}^{N} \sum_{l=1}^{n}\left[2 \gamma_{k}^{\frac{1-\theta}{1+\theta}} \frac{\gamma_{k}\left|\alpha_{k l}(t)\right|^{2}}{2}\right]^{\frac{\theta+1}{2}}
\end{aligned}
$$




$$
\begin{aligned}
& \leq-2^{\frac{\theta+1}{2}}\left(\widehat{\gamma}_{\min }^{\frac{1-\theta}{1+\theta}}\right)^{\frac{1+\theta}{2}}\left[\sum_{k=1}^{N} \sum_{l=1}^{n} \frac{\gamma_{k}}{2}\left|\alpha_{k l}(t)\right|^{2}\right]^{\frac{\theta+1}{2}} \\
& =-2^{\frac{\theta+1}{2}} \widehat{\gamma}_{\min }^{\frac{1-\theta}{2}} X^{\frac{\theta+1}{2}}(t) .
\end{aligned}
$$

From (22), we have

$$
-\xi \sum_{k=1}^{N} \sum_{l=1}^{n} \gamma_{k}\left|\alpha_{k l}(t)\right|^{\theta+1} \leq-\delta X^{\frac{1+\theta}{2}}(t)
$$

It follows from (14), (20), and (23) that

$$
D^{h} X(t) \leq-\delta X^{\frac{1+\theta}{2}}(t)
$$

According to Lemma 2.8, one has

$$
X^{\frac{2 h-\theta-1}{2}}(t) \leq X^{\frac{2 h-\theta-1}{2}}\left(t_{0}\right)-\frac{\delta \Gamma\left(\frac{2 h+1-\theta}{2}\right)\left(t-t_{0}\right)^{h}}{\Gamma(1+h) \Gamma\left(\frac{1-\theta}{2}\right)}, \quad t \in\left[t_{0}, t_{1}\right],
$$

and $X(t)=0, \forall t \geq t_{1}$, where $t_{1}$ is denoted by

$$
t_{1}=t_{0}+\left[\frac{\Gamma(1+h) \Gamma\left(\frac{1-\theta}{2}\right) X^{\frac{2 h-1-\theta}{2}}\left(t_{0}\right)}{\delta \Gamma\left(\frac{2 h+1-\theta}{2}\right)}\right]^{\frac{1}{h}} .
$$

Based on Definition 2.5, FCDNNs (1) and the isolated networks (2) are finite-time synchronized under the discontinuous feedback control protocol (4).

The following kinds of fractional-order coupled neural networks (FCNNs) are also a very interesting problem. The following assumption is needed to derive the FTS criterion of FCNNs with continuous activations.

Assumption $\left[\mathcal{H}_{4}\right]$. For every $k=1,2, \ldots, N, l=1,2, \ldots, n$, there exist positive constants $b_{l}>0$ such that, for every $p_{k l}(t) \in \mathbb{R}, q_{l}(t) \in \mathbb{R}$, the following inequality holds:

$$
\left|f_{k l}(t)-q_{l}(t)\right| \leq b_{l}\left|p_{k l}(t)-q_{l}(t)\right|
$$

Corollary 3.2 Suppose that Assumption $\left[\mathcal{H}_{4}\right]$ and conditions (7) and (8) of Theorem 3.1 hold. Then FCDNNs (1) and the isolated networks (2) with continuous activations are finite-time synchronized under the following feedback control protocol:

$$
y_{k}(t)=-\beta \alpha_{k}(t)-\zeta\left\|\alpha_{k}(t-\tau)\right\|_{1} \operatorname{sign}\left(\alpha_{k}(t)\right)-\xi \operatorname{sign}\left(\alpha_{k}(t)\right)\left|\alpha_{k}(t)\right|^{\theta},
$$

where $0<\theta<1, \beta>0, \zeta>0, \xi>0$ are control gains, and $\gamma_{k}$ signifies the cofactor of the ith diagonal elements of $\mathcal{L}$.

Proof The proof of the corollary is similar to that of Theorem 3.1. Hence the proof is omitted here. 


\subsection{Finite-time synchronization under discontinuous adaptive feedback control} Next, we design discontinuous adaptive feedback control protocol as follows:

$$
\left\{\begin{aligned}
y_{k}(t)= & -\beta_{k}(t) \alpha_{k}(t)-\zeta\left\|\alpha_{k}(t-\tau)\right\|_{1} \operatorname{sign}\left(\alpha_{k}(t)\right) \\
& -\left(\xi_{k}(t)+\eta\right) \operatorname{sign}\left(\alpha_{k}(t)\right) \\
D^{h} \beta_{k}(t) & =\lambda\left|\alpha_{k}(t)\right|^{2}-\phi \operatorname{sign}\left[\beta_{k}(t)-\beta\right]+\frac{\varpi\left|\alpha_{k}(t)\right|}{\left[\beta_{k}(t)-\beta\right]} \\
D^{h} \xi_{k}(t) & =\mu\left|\alpha_{k}(t)\right|-\sigma \operatorname{sgn}\left(\xi_{k}(t)-\chi\right)
\end{aligned}\right.
$$

where $\zeta>0, \eta>0, \lambda>0, \mu>0, \sigma>0, \beta>0, \varpi>0, \chi>0$ are all constants, $\beta_{k}(t)$ and $\xi_{k}(t)$ are adaptive control gains. According to the Filippov framework and set-valued map analysis, FCDNNs (3) can be written as follows:

$$
\begin{aligned}
D^{h} \alpha_{k}(t) \in & -U \alpha_{k}(t)+V \tilde{c o}\left\{\Psi\left(\alpha_{k}(t)\right)\right\}+W \tilde{c o}\left\{\Psi\left(\alpha_{k}(t-\tau)\right)\right\} \\
& +\sum_{l=1}^{N} a_{k l} E\left(\alpha_{l}(t)-\alpha_{k}(t)\right)-\zeta\left\|\alpha_{k}(t-\tau)\right\|_{1} \tilde{c}\left(\operatorname{sign}\left(\alpha_{k}(t)\right)\right) \\
& -\beta_{k}(t) \alpha_{k}(t)-\left(\xi_{k}(t)+\eta\right) \tilde{c o}\left(\operatorname{sign}\left(\alpha_{k}(t)\right)\right) \\
\subseteq & -U \alpha_{k}(t)+V\left[\tilde{c o}\left(p_{k}(t)\right)-\tilde{c o}(q(t))\right] \\
& +W\left[\tilde{c o}\left(p_{k}(t-\tau)\right)-\tilde{c o}(q(t-\tau))\right] \\
& +\sum_{l=1}^{N} a_{k l} E\left(\alpha_{l}(t)-\alpha_{k}(t)\right)-\beta_{k}(t) \alpha_{k}(t) \\
& -\zeta\left\|\alpha_{k}(t-\tau)\right\|_{1} \tilde{c o}\left(\operatorname{sign}\left(\alpha_{k}(t)\right)\right) \\
& -\left(\xi_{k}(t)+\eta\right)\left(\operatorname{sign}\left(\alpha_{k}(t)\right)\right) .
\end{aligned}
$$

Then there exist a measurable function $\tilde{\mu}_{k}(t) \in \tilde{c o}\left[f\left(p_{k}(t)\right)\right]$ and $\mu(t) \in \tilde{c o}[f(q(t))]$ for a.e. $t \in\left[-\tau, t_{1}\right]$ such that

$$
\begin{aligned}
D^{h} \alpha_{k}(t)= & -U \alpha_{k}(t)+V\left[\tilde{\mu}_{k}(t)-\mu(t)\right]+W\left[\tilde{\mu}_{k}(t-\tau)-\mu(t-\tau)\right] \\
& +\sum_{l=1}^{N} a_{k l} E\left(\alpha_{l}(t)-\alpha_{k}(t)\right)-\beta_{k}(t) \alpha_{k}(t) \\
& -\zeta\left\|\alpha_{k}(t-\tau)\right\|_{1} \operatorname{SIGN}\left(\alpha_{k}(t)\right)-\left(\xi_{k}(t)+\eta\right)\left(\operatorname{SIGN}\left(\alpha_{k}(t)\right)\right) .
\end{aligned}
$$

Theorem 3.3 Suppose that Assumptions $\left[\mathcal{H}_{1}\right]-\left[\mathcal{H}_{3}\right]$ and conditions (7)-(9) of Theorem 3.1 hold. Then FCDNNs (1) and the isolated networks (2) are finite-time synchronized under the discontinuous adaptive feedback control protocol (25), if

$$
\chi=1+\frac{\varpi}{\lambda} .
$$

Furthermore, the settling time is evaluated as follows:

$$
t_{1}=t_{0}+\left[\frac{X^{\frac{1}{2}}\left(t_{0}\right) \Gamma(1+h) \Gamma\left(\frac{1}{2}\right)}{\delta \Gamma\left(\frac{2 h+1}{2}\right)}\right]^{\frac{1}{h}},
$$


where

$$
\begin{aligned}
& X\left(t_{0}\right)=\sum_{k=1}^{N} \frac{\gamma_{k}}{2} \alpha_{k}^{T}\left(t_{0}\right) \alpha_{k}\left(t_{0}\right)+\sum_{k=1}^{N} \frac{\gamma_{k}}{2 \lambda}\left[\beta_{k}\left(t_{0}\right)-\beta\right]^{2}+\sum_{k=1}^{N} \frac{\gamma_{k}}{2 \mu}\left[\xi_{k}\left(t_{0}\right)-\chi\right]^{2}, \\
& \delta=\sqrt{2} \min \left\{\min \left\{\sqrt{\gamma_{k}}\right\}, \min \left\{\frac{\sqrt{\gamma_{k} \phi}}{\sqrt{\lambda}}\right\}, \min \left\{\frac{\sqrt{\gamma_{k} \sigma}}{\sqrt{\mu}}\right\}\right\},
\end{aligned}
$$

and $\gamma_{k}$ signifies the cofactor of the ith diagonal elements of $\mathcal{L}$.

Proof For the FCDNNs error system (6), construct the following Lyapunov functional:

$$
X(t)=\sum_{k=1}^{N} \frac{\gamma_{k}}{2} \alpha_{k}^{T}(t) \alpha_{k}(t)+\sum_{k=1}^{N} \frac{\gamma_{k}}{2 \lambda}\left[\beta_{k}(t)-\beta\right]^{2}+\sum_{k=1}^{N} \frac{\gamma_{k}}{2 \mu}\left[\xi_{k}(t)-\chi\right]^{2} .
$$

Since $(\mathcal{G}, A)$ is strongly connected, by using Lemma 2.1 that $\gamma_{k}>0$ for $k \in\{1,2, \ldots, N\}$, and Lemma 2.6, one can get

$$
\begin{aligned}
D^{h} X(t) \leq & \sum_{k=1}^{N} \gamma_{k} \alpha_{k}^{T}(t) D^{h}\left\{\alpha_{k}(t)\right\} \\
& +\sum_{k=1}^{N} \frac{\gamma_{k}}{\lambda}\left[\beta_{k}(t)-\beta\right] D^{h} \beta_{k}(t)+\sum_{k=1}^{N} \frac{\gamma_{k}}{\mu}\left[\xi_{k}(t)-\chi\right] D^{h} \xi_{k}(t) \\
= & \sum_{k=1}^{N} \gamma_{k} \alpha_{k}^{T}(t)\left[-U \alpha_{k}(t)+V\left[\tilde{\mu}_{k}(t)-\mu(t)\right]\right. \\
& +W\left[\tilde{\mu}_{k}(t-\tau)-\mu(t-\tau)\right]+\sum_{l=1}^{N} a_{k l} E\left(\alpha_{l}(t)-\alpha_{k}(t)\right) \\
& -\beta_{k}(t) \alpha_{k}(t)-\zeta\left\|\alpha_{k}(t-\tau)\right\| \|_{1} \operatorname{SIGN}\left(\alpha_{k}(t)\right) \\
& \left.-\left(\xi_{k}(t)+\eta\right)\left(\operatorname{SIGN}\left(\alpha_{k}(t)\right)\right)\right]+\sum_{k=1}^{N} \frac{\gamma_{k}}{\lambda}\left[\beta_{k}(t)-\beta\right] \\
& \times\left[\lambda\left|\alpha_{k}(t)\right|^{2}-\phi \operatorname{sign}\left[\beta_{k}(t)-\beta\right]+\frac{\varpi\left|\alpha_{k}(t)\right|}{\left[\beta_{k}(t)-\beta\right]}\right] \\
& +\sum_{k=1}^{N} \frac{\gamma_{k}}{\mu}\left[\xi_{k}(t)-\chi\right]\left[\mu\left|\alpha_{k}(t)\right|-\sigma \operatorname{sgn}\left(\xi_{k}(t)-\chi\right)\right] .
\end{aligned}
$$

By using Theorem 3.1, Eq. (12), and Eq. (10), one has

$$
\begin{aligned}
D^{h} X(t) \leq & -\left[\Lambda_{\min }(U)-n b^{\max } v^{\max }\right] \sum_{k=1}^{N} \gamma_{k} \alpha_{k}^{T}(t) \alpha_{k}(t) \\
& -\left[\zeta-b^{\max } w^{\max }\right] \sum_{k=1}^{N} \gamma_{k}\left\|\alpha_{k}(t)\right\|_{1}\left\|\alpha_{k}(t-\tau)\right\|_{1} \\
& -\left[\eta-n d^{\max }\left(v^{\max }+w^{\max }\right)\right] \sum_{k=1}^{N} \gamma_{k}\left\|\alpha_{k}(t)\right\|_{1}
\end{aligned}
$$




$$
\begin{aligned}
& -\sum_{k=1}^{N} \gamma_{k} \beta_{k}(t)\left|\alpha_{k}(t)\right|^{2}-\sum_{k=1}^{N} \gamma_{k} \xi_{k}(t)\left|\alpha_{k}(t)\right| \\
& +\sum_{k=1}^{N} \gamma_{k}\left[\beta_{k}(t)-\beta\right]\left|\alpha_{k}(t)\right|^{2}-\sum_{k=1}^{N} \frac{\gamma_{k}\left|\beta_{k}(t)-\beta\right| \phi}{\lambda} \\
& +\sum_{k=1}^{N} \frac{\gamma_{k}\left|\alpha_{k}(t)\right| \varpi}{\lambda}-\sum_{k=1}^{N} \frac{\gamma_{k}\left|\xi_{k}(t)-\chi\right| \sigma}{\mu} \\
& +\sum_{k=1}^{N} \gamma_{k}\left[\xi_{k}(t)-\chi\right]\left|\alpha_{k}(t)\right| \\
\leq & -\left[\Lambda_{\min }(U)+\beta-n b^{\max } v^{\max }\right] X(t) \\
& -\left[\zeta-b^{\max } w^{\max }\right] \sum_{k=1}^{N} \gamma_{k}\left\|\alpha_{k}(t)\right\|_{1}\left\|\alpha_{k}(t-\tau)\right\|_{1} \\
& -\left[\eta-n d^{\max }\left(v^{\max }+w^{\max }\right)\right] \sum_{k=1}^{N} \gamma_{k}\left\|\alpha_{k}(t)\right\|_{1} \\
& +\sum_{k=1}^{N} \gamma_{k}\left[\frac{\varpi}{\lambda}-\chi\right]\left|\alpha_{k}(t)\right|-\sum_{k=1}^{N} \frac{\gamma_{k}\left|\beta_{k}(t)-\beta\right| \phi}{\lambda} \\
& -\sum_{k=1}^{N} \frac{\gamma_{k}\left|\xi_{k}(t)-\chi\right| \sigma}{\mu} .
\end{aligned}
$$

By virtue of Lemma 2.7, we obtain

$$
\begin{aligned}
& \sqrt{\sum_{k=1}^{N} \gamma_{k}\left(\frac{1}{2}\left|\alpha_{k}(t)\right|^{2}+\frac{1}{2 \lambda}\left[\beta_{k}(t)-\beta\right]^{2}+\frac{1}{2 \mu}\left[\xi_{k}(t)-\chi\right]^{2}\right)} \\
& \leq \sum_{k=1}^{N} \sqrt{\gamma_{k}}\left(\frac{1}{\sqrt{2}}\left|\alpha_{k}(t)\right|+\frac{1}{\sqrt{2 \lambda}}\left[\beta_{k}(t)-\beta\right]+\frac{1}{\sqrt{2 \mu}}\left[\xi_{k}(t)-\chi\right]\right) .
\end{aligned}
$$

Then, from Eq. (7)-Eq. (9), Eq. (20), and Eq. (30)-Eq. (31), we can obtain

$$
\begin{aligned}
D^{h} X(t) \leq & -\sum_{k=1}^{N} \gamma_{k}\left[\chi-\frac{\varpi}{\lambda}\right]\left|\alpha_{k}(t)\right| \\
& -\sum_{k=1}^{N} \frac{\gamma_{k}\left|\beta_{k}(t)-\beta\right| \phi}{\lambda}-\sum_{k=1}^{N} \frac{\gamma_{k}\left|\xi_{k}(t)-\chi\right| \sigma}{\mu} \\
\leq & -2\left[\sum_{k=1}^{N} \frac{\gamma_{k}}{2}\left|\alpha_{k}(t)\right|+\sum_{k=1}^{N} \frac{\gamma_{k}\left|\beta_{k}(t)-\beta\right| \phi}{2 \lambda}+\sum_{k=1}^{N} \frac{\gamma_{k}\left|\xi_{k}(t)-\chi\right| \sigma}{2 \mu}\right] \\
\leq & -\delta X^{\frac{1}{2}}(t) .
\end{aligned}
$$

According to Lemma 2.8, one has

$$
X^{\frac{2 h-1}{2}}(t) \leq X^{\frac{1}{2}}\left(t_{0}\right)-\frac{\delta \Gamma\left(\frac{2 h+1}{2}\right)\left(t-t_{0}\right)^{h}}{\Gamma(1+h) \Gamma\left(\frac{1}{2}\right)}, \quad t \in\left[t_{0}, t_{1}\right] .
$$


The settling time $t_{1}$ is estimated by

$$
t_{1}=t_{0}+\left[\frac{X^{\frac{1}{2}}\left(t_{0}\right) \Gamma(1+h) \Gamma\left(\frac{1}{2}\right)}{\delta \Gamma\left(\frac{2 h+1}{2}\right)}\right]^{\frac{1}{h}} .
$$

Based on Definition 2.5, FCDNNs (1) and the isolated networks (2) are synchronized in finite time under the discontinuous adaptive feedback control protocol (25).

Corollary 3.4 Suppose that Assumption $\left[\mathcal{H}_{4}\right]$ and conditions (7), (8), and (28) of Theorem 3.3 hold. Then system (1) and the isolated networks (2) with continuous activations are finite-time synchronized under the following adaptive feedback control protocol:

$$
\left\{\begin{array}{l}
y_{k}(t)=-\beta_{k}(t) \alpha_{k}(t)-\zeta\left\|\alpha_{k}(t-\tau)\right\|_{1} \operatorname{sign}\left(\alpha_{k}(t)\right)-\xi_{k}(t) \operatorname{sign}\left(\alpha_{k}(t)\right), \\
D^{h} \beta_{k}(t)=\lambda\left|\alpha_{k}(t)\right|^{2}-\phi \operatorname{sign}\left[\beta_{k}(t)-\beta\right]+\frac{\varpi\left|\alpha_{k}(t)\right|}{\left[\beta_{k}(t)-\beta\right]} \\
D^{h} \xi_{k}(t)=\mu\left|\alpha_{k}(t)\right|-\sigma \operatorname{sgn}\left(\xi_{k}(t)-\chi\right),
\end{array}\right.
$$

where $\zeta>0, \lambda>0, \mu>0, \sigma>0, \beta>0, \varpi>0, \chi>0$ are all constants, $\beta_{k}(t)$ and $\xi_{k}(t)$ are adaptive control gains, and $\gamma_{k}$ signifies the cofactor of the ith diagonal elements of $\mathcal{L}$.

Proof The proof of the corollary is similar to that of Theorem 3.3. Hence the proof is omitted here.

Remark 3.5 It is the first time that the finite-time synchronization and adaptive finite-time synchronization criterion of FCDNNs have been investigated. In this paper, fractional order, discontinuations neuron activation, graph theory techniques, and coupling terms are taken into consideration, and their results are very complicated and not easy to calculate. The main innovation of this paper is to extend and to overcome this complication. Hence our proposed models are more general and advanced.

\section{Computer simulations}

Here, two numerical cases are given to illustrate the efficiency of the proposed finite-time synchronization results.

Example 4.1 Consider a class of FCDNNs on a directed graph $\mathbb{G}$ consisting of six identical nodes with every isolated node network being a 2-dimensional dynamical system, which is characterized by

$$
\begin{aligned}
D^{h} p_{k}(t)= & -U p_{k}(t)+V f\left(p_{k}(t)\right)+W f\left(p_{k}(t-\tau)\right) \\
& +\sum_{l=1}^{6} a_{k l} E\left(q_{l}(t)-q_{k}(t)\right)+y_{k}(t)
\end{aligned}
$$

with the isolated networks

$$
D^{h} p_{k}(t)=-U p_{k}(t)+V f\left(p_{k}(t)\right)+W f\left(p_{k}(t-\tau)\right),
$$


Figure 1 A directed graph $\mathbb{G}$ consisting of 6 identical nodes

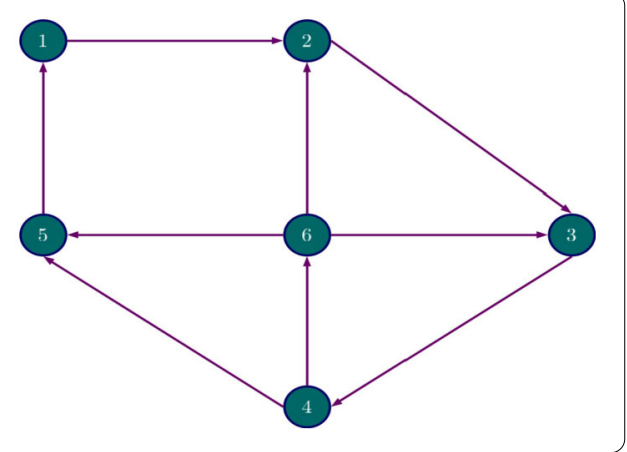

where $k=1,2,3,4,5,6, h=0.98$, let $U=\operatorname{diag}\{12,12\}, \tau=2.5, E=\operatorname{diag}\{1,1\}$, and

$$
V=\left[\begin{array}{cc}
0.5 & 2.8 \\
1.4 & -1.3
\end{array}\right], \quad W=\left[\begin{array}{cc}
0.6 & 1 \\
1.3 & -1.5
\end{array}\right] \text {. }
$$

From Fig. 1, we can see that the coupling matrix $A$ and the corresponding Laplacian matrix $\mathcal{L}(\mathbb{G}, A)$, respectively, are given as follows:

$$
A=\left[\begin{array}{llllll}
0 & 0 & 0 & 0 & 1 & 0 \\
1 & 0 & 0 & 0 & 0 & 1 \\
0 & 1 & 0 & 0 & 0 & 1 \\
0 & 0 & 1 & 0 & 0 & 0 \\
0 & 0 & 0 & 1 & 0 & 1 \\
0 & 0 & 0 & 1 & 0 & 0
\end{array}\right], \quad \mathcal{L}(\mathbb{G}, A)=\left[\begin{array}{cccccc}
1 & 0 & 0 & 0 & -1 & 0 \\
-1 & 2 & 0 & 0 & 0 & -1 \\
0 & -1 & 2 & 0 & 0 & -1 \\
0 & 0 & -1 & 1 & 0 & 0 \\
0 & 0 & 0 & -1 & 2 & -1 \\
0 & 0 & 0 & -1 & 0 & 1
\end{array}\right] .
$$

The discontinuous neuron activation is selected as $f(p)=0.5+\operatorname{sign}(p)$. Then one can obtain that $b^{\max }=1, d^{\max }=0.5$. The initial conditions are chosen as follows:

$$
\begin{aligned}
& p_{1}(0)=\left(p_{11}(0), p_{12}(0)\right)^{T}=(4,-4)^{T}, \\
& p_{2}(0)=\left(p_{21}(0), p_{22}(0)\right)^{T}=(2.5,-2.5)^{T}, \\
& p_{3}(0)=\left(p_{31}(0), p_{32}(0)\right)^{T}=(1.3,-3.5)^{T}, \\
& p_{4}(0)=\left(p_{41}(0), p_{42}(0)\right)^{T}=(1.25,-1.2)^{T}, \\
& p_{5}(0)=\left(p_{51}(0), p_{52}(0)\right)^{T}=(-4.25,3.25)^{T}, \\
& p_{6}(0)=\left(p_{61}(0), p_{62}(0)\right)^{T}=(2.7,-4.8)^{T}, \\
& q(0)=\left(q_{1}(0), q_{2}(0)\right)^{T}=(2.5,-4.5)^{T} .
\end{aligned}
$$

By simple calculation, we get $\gamma_{1}=2, \gamma_{2}=2, \gamma_{3}=4, \gamma_{4}=2, \gamma_{5}=1, \gamma_{6}=9$. The discontinuous feedback control protocol is designed by

$$
\begin{aligned}
y_{k}(t)= & -14 \alpha_{k}(t)-3\left\|\alpha_{k}(t-\tau)\right\|_{1} \operatorname{sign}\left(\alpha_{k}(t)\right) \\
& -6 \operatorname{sign}\left(\alpha_{k}(t)\right)-0.6 \operatorname{sign}\left(\alpha_{k}(t)\right)\left|\alpha_{k}(t)\right|^{0.6}
\end{aligned}
$$


Figure 2 The state trajectories of $q_{1}(t)$ vs. $p_{k 1}(t)$ for $k=1,2,3,4,5,6$ with control (37)

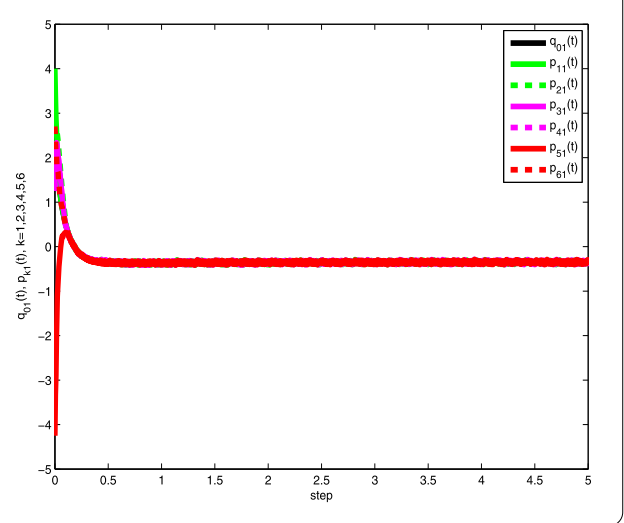

for $k=1,2,3,4,5,6$. According to Theorem 3.1, it is simple to check

$$
\begin{aligned}
& 14=\beta>\Lambda_{\min }(U)-n b^{\max } v^{\max }=6.4, \\
& 3=\zeta>b^{\max } w^{\max }=1.5, \\
& 6=\eta>n d^{\max }\left(v^{\max }+w^{\max }\right)=4.3,
\end{aligned}
$$

and all the conditions of Theorem 3.1 hold. Therefore, FCDNNs (35) and the isolated networks (36) are finite-time synchronized under the discontinuous feedback control proto$\operatorname{col}(37)$.

Furthermore, the settling time is evaluated as follows:

$$
\begin{aligned}
{[H] t_{1} } & =t_{0}+\left[\frac{\Gamma(1+h) \Gamma\left(\frac{1-\theta}{2}\right) X^{\frac{2 h-1-\theta}{2}}\left(t_{0}\right)}{\delta \Gamma\left(\frac{2 h+1-\theta}{2}\right)}\right]^{\frac{1}{h}} \\
& =0+\left[\frac{\Gamma(1+0.98) \Gamma\left(\frac{1-0.7}{2}\right) X^{\frac{2(0.98)-1-0.9}{2}}(0)}{1.0815 \times \Gamma\left(\frac{2(0.98)+1-0.7}{2}\right)}\right]^{\frac{1}{0.98}} \\
& =\left[\frac{\Gamma(1.98) \Gamma(0.15)(18.595)^{0.13}}{1.0815 \times \Gamma(1.13)}\right]^{\frac{1}{0.98}} \\
& =8.916 .
\end{aligned}
$$

Figures 2-5 demonstrate the numerical simulation results, which confirms the obtained theoretical findings. Figure 2 and Fig. 3 display the time response of considered systems (35) and (36) with control input (37), respectively, while the time response of synchronization errors $\alpha_{k 1}(t)$ and $\alpha_{k 2}(t)$ is displayed in Fig. 4 and Fig. 5, respectively.

Example 4.2 Consider a class of FCDNNs on directed graph $\mathbb{G}$ consisting of four identical nodes characterized by the following form:

$$
\begin{aligned}
D^{h} p_{k}(t)= & -U p_{k}(t)+V f\left(p_{k}(t)\right) \\
& +W f\left(p_{k}(t-\tau)\right)+\sum_{l=1}^{4} a_{k l} E\left(q_{l}(t)-q_{k}(t)\right)+y_{k}(t)
\end{aligned}
$$


Figure 3 The state trajectories of $q_{2}(t)$ vs. $p_{k 2}(t)$ for $k=1,2,3,4,5,6$ with control (37)

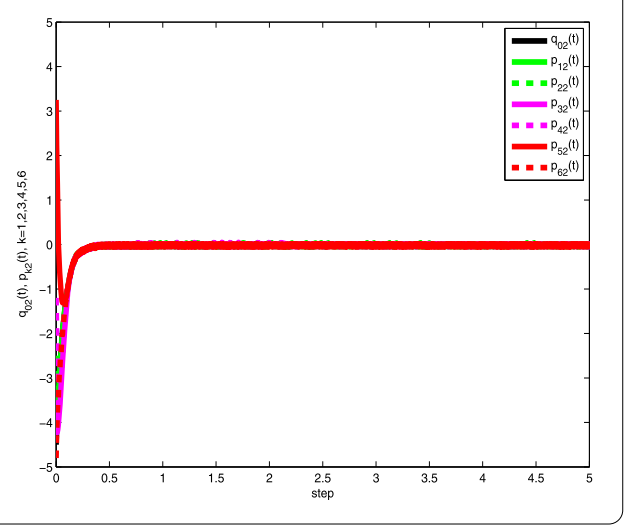

Figure 4 The change processes of synchronization errors with control (37)

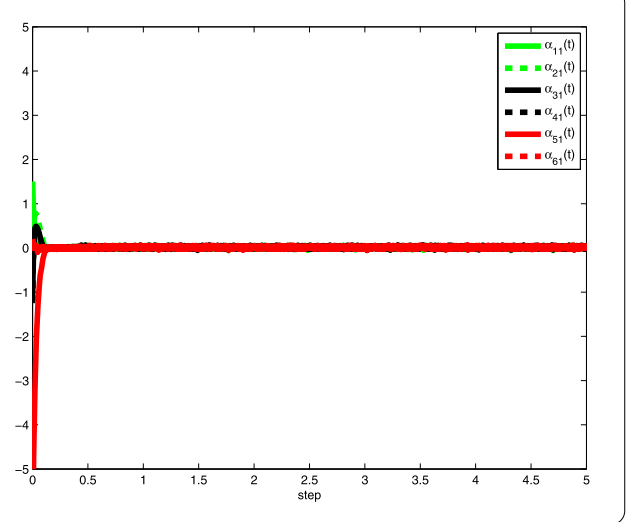

Figure 5 The change processes of synchronization errors with control (37)

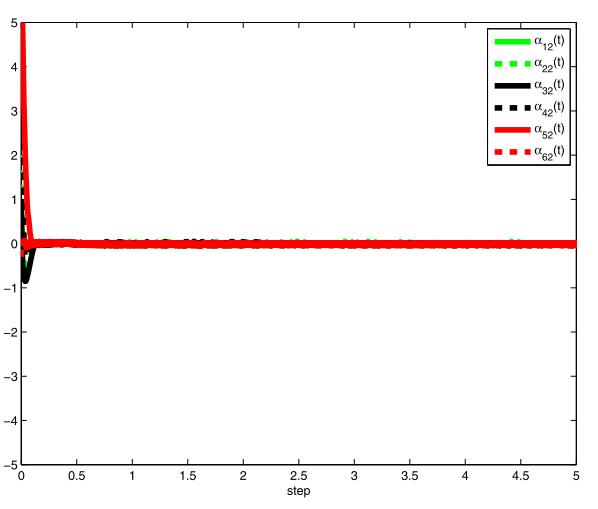

with the isolated networks

$$
D^{h} p_{k}(t)=-U p_{k}(t)+V f\left(p_{k}(t)\right)+W f\left(p_{k}(t-\tau)\right),
$$

where $k=1,2,3,4, h=0.98$, let $U=\operatorname{diag}\{10,10\}, \tau=1.5, E=\operatorname{diag}\{1,1\}$, and

$$
V=\left[\begin{array}{cc}
1.4 & -0.4 \\
-2.2 & 1.2
\end{array}\right], \quad W=\left[\begin{array}{cc}
1 & 1 \\
-1 & 1.5
\end{array}\right]
$$


Figure 6 A directed graph $\mathbb{G}$ consisting of 4 identical nodes

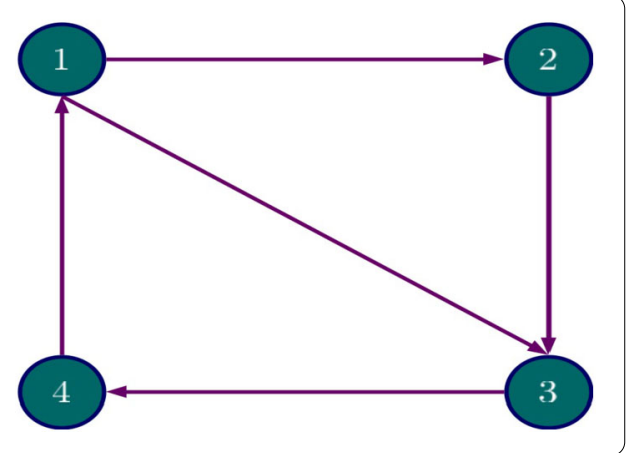

Figure 7 The state trajectories of $q_{1}(t)$ vs. $p_{k 1}(t)$ for $k=1,2,3,4$ with control (40)

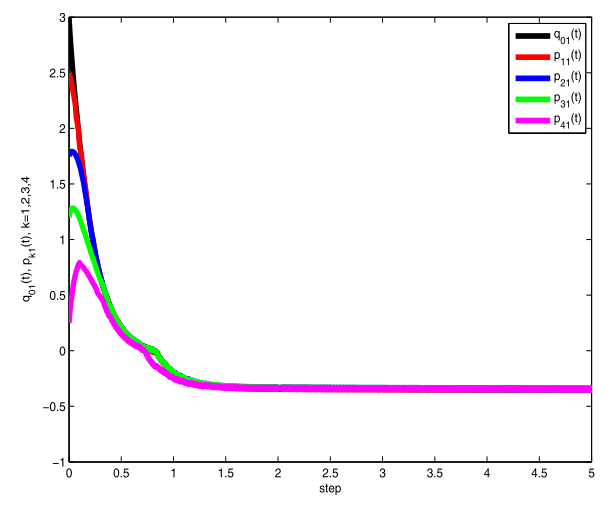

From Fig. 6, we can see that the coupling matrix $A$ and the corresponding Laplacian matrix $\mathcal{L}(\mathbb{G}, A)$, respectively, are given as follows:

$$
A=\left[\begin{array}{cccccc}
0 & 0 & 0 & 0 & 1 & 0 \\
1 & 0 & 0 & 0 & 0 & 1 \\
0 & 1 & 0 & 0 & 0 & 1 \\
0 & 0 & 1 & 0 & 0 & 0 \\
0 & 0 & 0 & 1 & 0 & 1 \\
0 & 0 & 0 & 1 & 0 & 0
\end{array}\right], \quad \mathcal{L}(\mathbb{G}, A)=\left[\begin{array}{cccccc}
1 & 0 & 0 & 0 & -1 & 0 \\
-1 & 2 & 0 & 0 & 0 & -1 \\
0 & -1 & 2 & 0 & 0 & -1 \\
0 & 0 & -1 & 1 & 0 & 0 \\
0 & 0 & 0 & -1 & 2 & -1 \\
0 & 0 & 0 & -1 & 0 & 1
\end{array}\right] .
$$

The discontinuous neuron activation is chosen as $f(p)=0.1+\operatorname{sign}(p)$. Then one can obtain that $b^{\max }=1.75, d^{\max }=0.2$. The initial conditions of systems (38) and (39) are: $p_{1}(0)=$ $(2.5,2.5), p_{2}(0)=(1.75,2.5)^{T}, p_{3}(0)=(1.2,1.5)^{T}, p_{4}(0)=(0.25,2)^{T}, q(0)=(3,3)^{T}$.

By simple calculation, we get $\gamma_{1}=2, \gamma_{2}=4, \gamma_{3}=2, \gamma_{4}=1$. The discontinuous adaptive feedback control protocol is designed by

$$
\left\{\begin{array}{l}
y_{k}(t)=-\beta_{k}(t) \alpha_{k}(t)-3\left\|\alpha_{k}(t-\tau)\right\|_{1} \operatorname{sign}\left(\alpha_{k}(t)\right) \\
\quad-\left(\xi_{k}(t)+2\right) \operatorname{sign}\left(\alpha_{k}(t)\right) \\
D^{h} \beta_{k}(t)=\left|\alpha_{k}(t)\right|^{2}-0.5 \operatorname{sign}\left[\beta_{k}(t)-2.5\right]+\frac{0.004\left|\alpha_{k}(t)\right|}{\left[\beta_{k}(t)-\beta\right]} \\
D^{h} \xi_{k}(t)=0.95\left|\alpha_{k}(t)\right|-\sigma \operatorname{sgn}\left(\xi_{k}(t)-1.004\right)
\end{array}\right.
$$




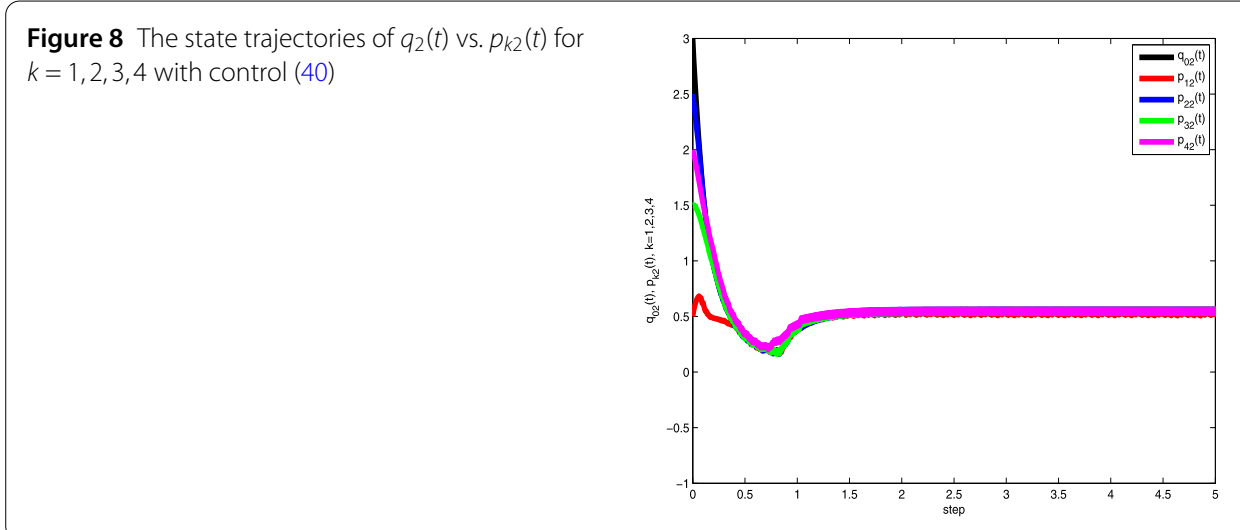

Figure 9 The change processes of synchronization errors with control (40)

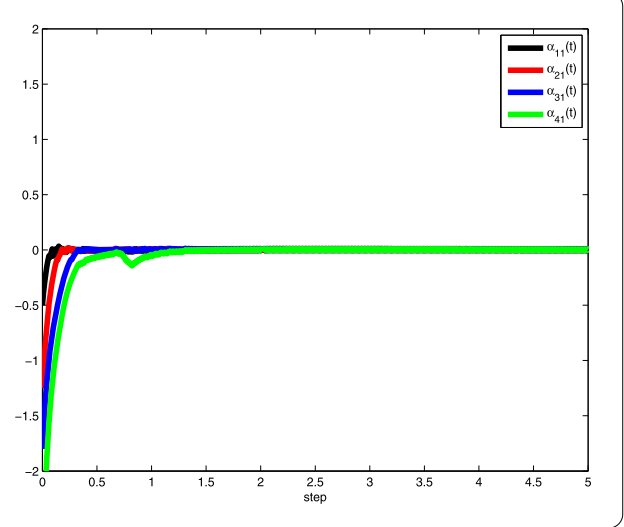

for $k=1,2,3,4$. According to Theorem 3.3, it is simple to check

$$
\begin{aligned}
& 2.5=\beta>\Lambda_{\min }(U)-n b^{\max } v^{\max }=2.3, \\
& 3=\zeta>b^{\max } w^{\max }=2.625, \\
& 2=\eta>n d^{\max }\left(v^{\max }+w^{\max }\right)=1.48,
\end{aligned}
$$

and all the conditions of Theorem 3.3 are satisfied. Therefore, FCDNNs (38) and the isolated networks (39) are finite-time synchronized under the discontinuous adaptive feedback control protocol (40). Next, we take the initial values of the discontinuous adaptive feedback control protocol (40) as follows: $\beta_{1}(0)=(0.02,0.03)^{T}, \beta_{2}(0)=(0.03,0.01)^{T}$, $\beta_{3}(0)=(0.02,0.01)^{T}, \beta_{4}(0)=(0.01,0.02)^{T}, \xi_{1}(0)=(0.05,0.03)^{T}, \xi_{2}(0)=(0.04,0.03)^{T}, \xi_{3}(0)=$ $(0.03,0.04)^{T}$, and $\xi_{4}(0)=(0.02,0.01)^{T}$.

Furthermore, the settling time is evaluated as follows:

$$
\begin{aligned}
t_{1} & =t_{1}=t_{0}+\left[\frac{X^{\frac{1}{2}}\left(t_{0}\right) \Gamma(1+h) \Gamma\left(\frac{1}{2}\right)}{\delta \Gamma\left(\frac{2 h+1}{2}\right)}\right]^{\frac{1}{h}} . \\
& =0+\left[\frac{\sqrt{76.945} \times \Gamma(1+0.98) \sqrt{\pi}}{1.414 \times \Gamma\left(\frac{2(0.98)+1}{2}\right)}\right]^{\frac{1}{0.98}} \\
& =12.95 .
\end{aligned}
$$


Figure 10 The change processes of synchronization errors with control (40)

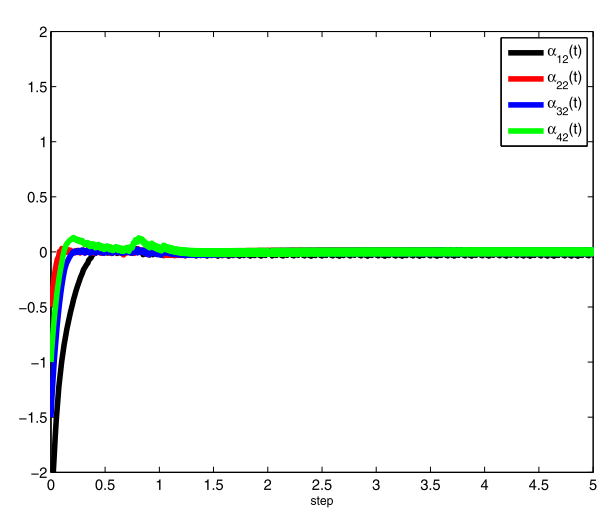

Figure 11 The evolutions of the control gains $\beta_{k}(t)(k=1,2,3,4)$ of controller $(40)$

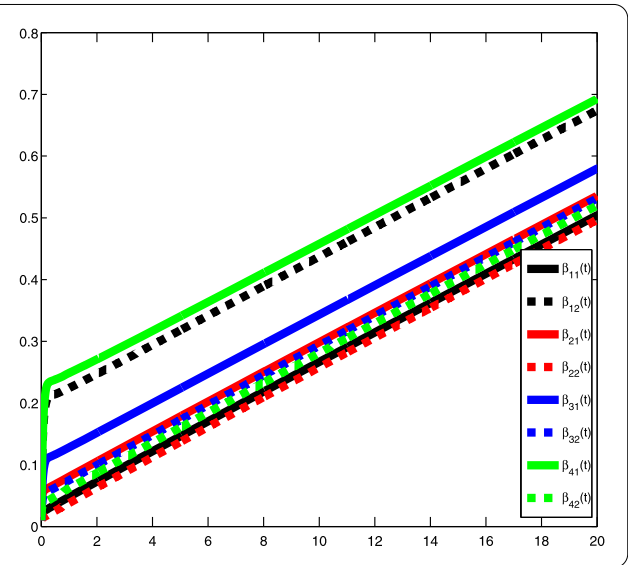

Figure 12 The evolutions of the control gains $\xi_{k}(t)$ $(k=1,2,3,4)$ of controller (40)

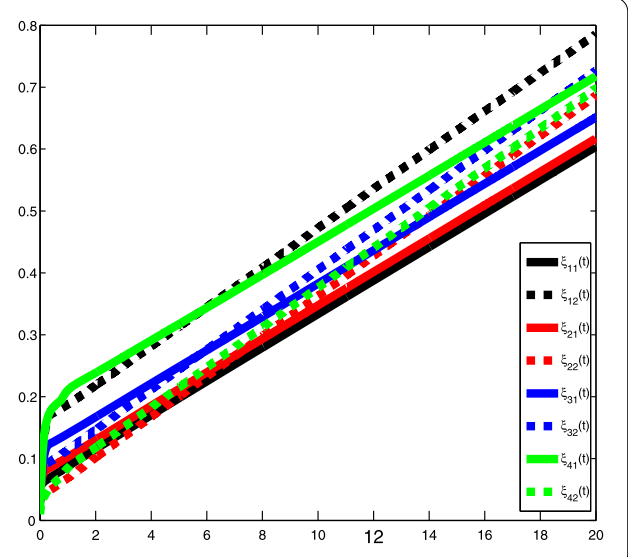

Figures 6-12 demonstrate the numerical simulation results, which confirms the accuracy of the theoretical results. Figure 7 and Fig. 8 show the state trajectories of considered systems (38) and (39) respectively. Figures 9-10 present the evaluations of synchronization errors between FCDNNs (38) and isolated networks (39) under the controller (40). Figure 11-12 demonstrate the adaptive feedback control gains (40), which shows that the adaptive control gains may go to some positive constants. 


\section{Conclusions}

In this research paper, we have examined the finite-time synchronization and adaptive finite-time synchronization for graph theory perspective FCDNNs. By employing differential inclusion theory, Filippov framework, and designed discontinuous controllers, several finite-time synchronization criteria are established based on the graph theory approach. Numerical computer simulations are given to illustrate the accuracy of the proposed finite-time synchronization results. Our future work will be focused on finite-time synchronization criterion for graph theory perspective FCDNNs with coupling delays and impulses.

\section{Acknowledgements}

The authors would like to thank three anonymous referees and the editor for very helpful suggestions and comments which led to improvements of our original paper.

\section{Funding}

This article has been written with the joint financial support of the National Natural Science Foundation of China (Nos. 11971076, 51839002), RUSA-Phase 2.0 Grant No. F 24-51/2014-U, Policy (TN Multi-Gen), Dept. of Edn. Govt. of India, UGC-SAP (DRS-I) Grant No. F.510/8/DRS-I/2016(SAP-I), and DST (FIST-level I) 657876570 Grant No. SR/FIST/MS-I/2018/17.

\section{Availability of data and materials}

Data sharing not applicable to this article as no datasets were generated or analysed during the current study.

\section{Competing interests}

The authors declare that they have no competing interests.

\section{Authors' contributions}

The authors contributed equally to this work. All authors read and approved the final manuscript.

\section{Author details}

${ }^{1}$ Vel Tech High Tech Dr Rangarajan Dr Sakunthala Engineering College, Avadi, India. ${ }^{2}$ Ramanujan Centre for Higher Mathematics, Alagappa University, Karaikudi, India. ${ }^{3}$ School of Mathematics, Southeast University, Nanjing, China. ${ }^{4}$ Department of Mathematics and General Sciences, Prince Sultan University, Riyadh, Saudi Arabia. ${ }^{5}$ School of Mathematics and Statistics, and Hunan Provincial Key Laboratory of Mathematical Modeling and Analysis in Engineering, Changsha University of Science and Technology, Changsha, China.

\section{Publisher's Note}

Springer Nature remains neutral with regard to jurisdictional claims in published maps and institutional affiliations.

Received: 19 December 2019 Accepted: 17 February 2020 Published online: 28 February 2020

\section{References}

1. Huang, C., Zhang, H., Cao, J., Hu, H.: Stability and Hopf bifurcation of a delayed prey-predator model with disease in the predator. Int. J. Bifurc. Chaos 29(7), 1950091 (2019)

2. Huang, C., Long, X., Huang, L., Fu, S.: Stability of almost periodic Nicholson's blowflies model involving patch structure and mortality terms. Can. Math. Bull. (2019). https://doi.org/10.4153/S0008439519000511

3. Long, X., Gong, S.: New results on stability of Nicholson's blowflies equation with multiple pairs of time-varying delays. Appl. Math. Lett. (2019). https://doi.org/10.1016/j.aml.2019.106027

4. Huang, C., Qiao, Y., Huang, L., Agarwal, R.: Dynamical behaviors of a food-chain model with stage structure and time delays. Adv. Differ. Equ. 2018, 186 (2018). https://doi.org/10.1186/s13662-018-1589-8

5. Hu, H., Zou, X.: Existence of an extinction wave in the Fisher equation with a shifting habitat. Proc. Am. Math. Soc. 145(11), 4763-4771 (2017)

6. Huang, C., Zhang, H., Huang, L.: Almost periodicity analysis for a delayed Nicholson's blowflies model with nonlinear density-dependent mortality term. Commun. Pure Appl. Anal. 18(6), 3337-3349 (2019)

7. Hu, H., Yi, T., Zou, X.: On spatial-temporal dynamics of Fisher-KPP equation with a shifting environment. Proc. Am. Math. Soc. 148(1), 213-221 (2020)

8. Yang, X., Wen, S., Liu, Z., Li, C., Huang, C.: Dynamic properties of foreign exchange complex network. Mathematics 7(9), 832 (2019). https://doi.org/10.3390/math7090832

9. Wang, J., Huang, C., Huang, L.: Discontinuity-induced limit cycles in a general planar piecewise linear system of saddle-focus type. Nonlinear Anal. Hybrid Syst. 33, 162-178 (2019)

10. Song, C., Fei, S., Cao, J., Huang, C.: Robust synchronization of fractional-order uncertain chaotic systems based on output feedback sliding mode control. Mathematics 7(7), 599 (2019). https://doi.org/10.3390/math7070599

11. Wang, J., Chen, X., Huang, L.: The number and stability of limit cycles for planar piecewise linear systems of node-saddle type. J. Math. Anal. Appl. 469(1), 405-427 (2019)

12. Zuo, Y., Wang, Y., Liu, X.: Adaptive robust control strategy for rhombus-type lunar exploration wheeled mobile robot using wavelet transform and probabilistic neural network. Comput. Appl. Math. 37, 314-337 (2018) 
13. Huang, C., Liu, B., Tian, X., Yang, L., Zhang, X.: Global convergence on asymptotically almost periodic SICNNs with nonlinear decay functions. Neural Process. Lett. 49(2), 625-641 (2019)

14. Liu, Y., Wu, J.: Fixed point theorems in piecewise continuous function spaces and applications to some nonlinear problems. Math. Methods Appl. Sci. 37(4), 508-517 (2014)

15. Cai, Z., Huang, J., Huang, L.: Generalized Lyapunov-Razumikhin method for retarded differential inclusions: applications to discontinuous neural networks. Discrete Contin. Dyn. Syst., Ser. B 22(9), 3591-3614 (2017)

16. Huang, C., Yang, Z., Yi, T., Zou, X.: On the basins of attraction for a class of delay differential equations with non-monotone bistable nonlinearities. J. Differ. Equ. 256(7), 2101-2114 (2014)

17. Huang, C., Cao, J., Wen, F., Yang, X.: Stability analysis of SIR model with distributed delay on complex networks. PLoS ONE 11(8), E0158813 (2016)

18. Tan, Y., Huang, C., Sun, B., Wang, T.: Dynamics of a class of delayed reaction-diffusion systems with Neumann boundary condition. J. Math. Anal. Appl. 458(2), 1115-1130 (2018)

19. Rajchakit, G., Pratap, A., Raja, R., Cao, J., Alzabut, J., Huang, C.: Hybrid control scheme for projective lag synchronization of Riemann-Liouville sense fractional order memristive BAM neural networks with mixed delays. Mathematics 7(8), 759 (2019). https://doi.org/10.3390/math7080759

20. Huang, C., Su, R., Cao, J., Xiao, S.: Asymptotically stable high-order neutral cellular neural networks with proportional delays and D operators. Math. Comput. Simul. 171, 127-135 (2020)

21. Cao, Y., Sriraman, R., Samidurai, R.: Stability and stabilization analysis of nonlinear time-delay systems with randomly occurring controller gain fluctuation. Math. Comput. Simul. 171, 36-51 (2020)

22. Cao, Y., Samidurai, R., Sriraman, R.: Stability and dissipativity analysis for neutral type stochastic Markovian jump static neural networks with time delays. J. Artif. Intell. Soft Comput. Res. 9(3), 189-204 (2019)

23. Yang, D., Li, X., Qiu, J.: Output tracking control of delayed switched systems via state-dependent switching and dynamic output feedback. Nonlinear Anal. Hybrid Syst. 32, 294-305 (2019)

24. Yang, X., Li, X., Xi, Q., Duan, P.: Review of stability and stabilization for impulsive delayed systems. Math. Biosci. Eng. 15(6), 1495-1515 (2018)

25. Li, X., Yang, X., Huang, T.: Persistence of delayed cooperative models: impulsive control method. Appl. Math. Comput. $342,130-146(2019)$

26. Hu, H., Yuan, X., Huang, L., Huang, C.: Global dynamics of an SIRS model with demographics and transfer from infectious to susceptible on heterogeneous networks. Math. Biosci. Eng. 16(5), 5729-5749 (2019)

27. Huang, C., Yang, X., Cao, J.: Stability analysis of Nicholson's blowflies equation with two different delays. Math. Comput. Simul. 171, 201-206 (2020)

28. Caponetto, R.: Fractional Order Systems: Modeling and Control Applications. World Scientific, Singapore (2010)

29. Podlubny, l.: Fractional Differential Equations. Academic Press, San Diego (1999)

30. Bagley, R.L., Torvik, P.J.: A theoretical basis for the application of fractional calculus to viscoelasticity. J. Rheol. 27(3), 201-210 (1983)

31. Garcia-Gonzalez, M.A., Fernández-Chimeno, M., Capdevila, L.: An application of fractional differintegration to heart rate variability time series. Comput. Methods Programs Biomed. 111(1), 33-40 (2013)

32. Miskinis, P.: Modelling linear reactions in inhomogeneous catalytic systems. J. Math. Chem. 51(13), 914-926 (2013)

33. Saptarshi, D., Indranil, P.: Fractional Order Signal Processing. Springer, Heidelberg (2012)

34. Suarez, J.I., Vinagre, B.M., Calderón, A., Monje, C.A., Chen, Y.Q.: Using fractional calculus for lateral and longitudinal control of autonomous vehicles in computer aided systems theory-EUROCAST. In: Lecture Notes in Computer Science, pp. 337-348. Springer, Berlin (2003)

35. Ding, X., Shen, Y.: Global dissipativity of fractional-order neural networks with time delays and discontinuous activations. Neurocomputing 196, 159-166 (2016)

36. Ding, X., Zeng, Z., Wang, L.: Robust finite-time stabilization of fractional-order neural networks with discontinuous and continuous activation functions under uncertainty. IEEE Trans. Neural Netw. Learn. Syst. 29(5), 1477-1490 (2018)

37. Liang, S., Wu, R., Chen, L.: Adaptive pinning synchronization in fractional-order uncertain complex dynamical networks with delay. Physica A 444, 49-62 (2015)

38. Ren, G., Yu, Y.: Pinning synchronization of fractional general complex dynamical networks with time Delay (2017). IFAC Papers Online 50-1 8058-8065

39. Bao, H., Cao, J.: Projective synchronization of fractional-order memristor-based neural networks. Neural Netw. 63, 1-9 (2015)

40. Pratap, A., Raja, R., Rajchakit, G., Cao, J., Bagdasar, O.: Mittag-Leffler state estimator design and synchronization analysis for fractional order BAM neural networks with time delays. Int. J. Adapt. Control Signal Process. 33(5), 855-874 (2019)

41. Stamova, I: Global Mittag-Leffler stability and synchronization of impulsive fractional order neural networks with time-varying delays. Nonlinear Dyn. 77, 1251-1260 (2014)

42. Yang, X., Huang, T., Song, Q., Huang, J.: Synchronization of fractional-order memristor-based complex-valued neural networks with uncertain parameters and time delays. Chaos Solitons Fractals 110, 105-123 (2018)

43. Yingjie, F., Xia, H., Zhen, W., Yuxia, L.: Global dissipativity and quasi-synchronization of asynchronous updating fractional-order memristor-based neural networks via interval matrix method. J. Franklin Inst. 355(13), 5998-6025 (2018)

44. Qian, C., Hu, Y:: Novel stability criteria on nonlinear density-dependent mortality Nicholson's blowflies systems in asymptotically almost periodic environments. J. Inequal. Appl. 2020, 13 (2020). https://doi.org/10.1186/s13660-019-2275-4

45. Cao, Q., Wang, G., Qian, C.: New results on global exponential stability for a periodic Nicholson's blowflies model involving time-varying delays. Adv. Differ. Equ. 2020, 43 (2020). https://doi.org/10.1186/s13662-020-2495-4

46. Cao, Q., Wang, G., Zhang, H., Gong, S.: New results on global asymptotic stability for a nonlinear density-dependent mortality Nicholson's blowflies model with multiple pairs of time-varying delays. J. Inequal. Appl. 2020, 13 (2020). https://doi.org/10.1186/s13660-019-2277-2

47. Manickam, I., Raja, R., Rajchakit, G., Cao, J., Alzabut, J., Huang, C.: A perspective on graph theory-based stability analysis of impulsive stochastic recurrent neural networks with time-varying delays. Adv. Differ. Equ. 2019, 502 (2019). https://doi.org/10.1186/s13662-019-2443-3 
48. Manickam, I., Raja, R., Rajchakit, G., Cao, J., Alzabut, J., Huang, C.: Existence, uniqueness and exponential stability of periodic solution for discrete-time delayed BAM neural networks based on coincidence degree theory and graph theoretic method. Mathematics 7(11), 1055 (2019). https://doi.org/10.3390/math7111055

49. Velmurugan, G., Rakkiyappan, R., Cao, J.: Finite-time synchronization of fractional-order memristor-based neural networks with time delays. Neural Netw. 73, 36-46 (2016)

50. Xiao, J., Zhong, S., Li, Y., Xu, F.: Finite-time Mittag-Leffler synchronization of fractional-order memristive BAM neural networks with time delays. Neurocomputing 219, 431-439 (2016)

51. Zhang, L., Yang, Y., Xu, X.: Synchronization analysis for fractional order memristive Cohen-Grossberg neural networks with state feedback and impulsive control. Physica A 506, 644-660 (2018)

52. Li, H., Cao, J., Jiang, H., Alsaedi, A.: Graph theory-based finite-time synchronization of fractional-order complex dynamical networks. J. Franklin Inst. 355(13), 5771-5789 (2018)

53. Li, H., Cao, J., Hu, C., Zhang, L., Wang, Z.: Global synchronization between two fractional-order complex networks with non-delayed and delayed coupling via hybrid impulsive control. Neurocomputing 356, 31-39 (2019)

54. Huang, C., Liu, B.: New studies on dynamic analysis of inertial neural networks involving non-reduced order method. Neurocomputing 325, 283-287 (2019)

55. Huang, C., Zhang, H.: Periodicity of non-autonomous inertial neural networks involving proportional delays and non-reduced order method. Int. J. Biomath. 12(2), 1950016 (2019)

56. Cao, J., Wang, Z., Sun, Y.: Synchronization in an array of linearly stochastically coupled networks with time delays. Phys. A, Stat. Mech. Appl. 385(2), 718-728 (2007)

57. Song, Q.: Synchronization analysis of coupled connected neural networks with mixed time delays. Neurocomputing 72(16-18), 3907-3914 (2009)

58. Yang, X., Cao, J., Lu, J.: Stochastic synchronization of coupled neural networks with intermittent control. Physica A 373(36), 3259-3272 (2009)

59. Cao, J., Li, L.: Cluster synchronization in an array of hybrid coupled neural networks with delay. Neural Netw. 22(4), 335-342 (2009)

60. Gray, C.M.: Synchronous oscillations in neuronal systems: mechanisms and functions. J. Comput. Neurosci. 1, 11-38 (1994)

61. Zhang, X.M., Sheng, S.Y., Lu, G.P., Zheng, Y.F.: Synchronization for arrays of coupled jumping delayed neural networks and its application to image encryption. In: Proceeding of the 556th Annual Conference on Decision and Control (2017)

62. Shuxue, W., Yanli, H., Shunyan, R.: Synchronization and robust synchronization for fractional-order coupled neural networks. IEEE Access 5, 12439-12448 (2017)

63. Zhang, H., Ye, M., Ye, R., Cao, J.: Synchronization stability of Riemann-Liouville fractional delay-coupled complex neural networks. Physica A 508(15), 155-165 (2018)

64. Li, M., Shuai, Z:: Global-stability problem for coupled systems of differential equations on networks. J. Differ. Equ. 248, $1-20(2010)$

65. Kilbas, A.A., Srivastava, H.M., Trujillo, J.J.: Theory and Applications of Fractional Differential Equations. Elsevier B.V Amsterdam (2006)

66. Filippov, A.F.: Differential Equations with Discontinuous Right-Hand Sides. Kluwer, Dordrecht (1988)

67. Aguila-Camacho, N., Duarte-Mermoud, M., Gallegos, J.: Lyapunov functions for fractional order systems. Commun Nonlinear Sci. Numer. Simul. 19, 2951-2957 (2014)

68. Hardy, G., Littlewood, J., Polya, G.: Inequalities. Cambridge University Press, Cambridge (1952)

69. Ding, Z., Shen, Y., Wang, L.: Global Mittag-Leffler synchronization of fractional order neural networks with discontinuous activations. Neural Netw. 73, 77-85 (2016)

70. Li, X., Fang, J., Zhang, W., Li, H.: Finite-time synchronization of fractional-order memristive recurrent neural networks with discontinuous activation functions. Neurocomputing 316(17), 284-293 (2018)

\section{Submit your manuscript to a SpringerOpen ${ }^{\circ}$ journal and benefit from:}

- Convenient online submission

- Rigorous peer review

- Open access: articles freely available online

- High visibility within the field

- Retaining the copyright to your article

Submit your next manuscript at $\gg$ springeropen.com 